Ann. Génét. Sél. anim., I977, 9 (4), 463-470.

\title{
Les anomalies chromosomiques des bovins (Bos taurus L.). État actuel des connaissances*
}

\author{
C. P. POPESCU \\ Laboratoire de Cytogénétique I.N.R.A.-U.N.C.E.I.A., \\ Centre national de Recherches zootechniques, I.N.R.A., \\ 78350 Jouy-en-Josas
}

\begin{abstract}
Résumé
Basé sur les données de la littérature et communiquées par les participants au III Colloque de Cytogénétique, a été établi un inventaire des anomalies chromosomiques des bovins. Treize mille animaux ayant fait l'objet d'une étude cytogénétique ont été repertoriés. Les tableaux présentent les anomalies de structure (tabl. r) et de nombre (tabl. 3) ainsi que la liste des 28 races dans lesquelles la translocation I/29 a été identifiée.
\end{abstract}

Les recherches sur les chromosomes des bovins domestiques ont été stimulées par la découverte d'une anomalie structurale, la translocation robertsonienne I /29 (Gustavsson et ROCKBORN, 1964). Les conséquences de cette anomalie sur la fer. tilité (Gustavsson, I969, ReFsdal, I976) ainsi que les risques de propagation accrus par l'insémination artificielle et par le transfert accéléré des populations animales, ont incité de nombreux pays à développer les études cytogénétiques des bovins.

Il nous a paru important d'inventorier les animaux étudiés dans différents laboratoires de cytogénétique et de dresser la liste des anomalies connues actuellement chez cette espèce.

Les trois tableaux présentés ici, contiennent les données recueillies dans la littérature, ainsi que les résultats non publiés, communiqués par les chercheurs réunis à l'occasion du III Colloque de Cytogénétique.

\section{L'effectif des animaux étudiés}

En considérant les données de la littérature, ainsi que celles fournies par différents laboratoires, nous avons recensé $\mathrm{I} 3000$ animaux appartenant à 80 races.

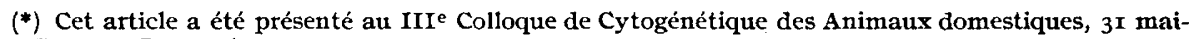
2 juin, Jouy-en-Josas, France. 





TABLEAU 2

La présence de la fusion I/29 dans différentes races bovines

\begin{tabular}{|c|c|c|c|c|c|c|}
\hline$N^{\prime \prime}$ & Race & & & & Pays & Auteurs \\
\hline $\mathbf{I}$ & Simmental . . . & & . . & & $\begin{array}{l}\text { Allemagne, Suisse, } \\
\text { Grande-Bretagne }\end{array}$ & $\begin{array}{l}\text { HERZOG et HÖHN (197r), POPESCU et al. } \\
\text { (r975), TSCHUDI, I977 (non publié), } \\
\text { HARVEY (I972) }\end{array}$ \\
\hline 2 & Holstein . . . . . & . & . . & & Suisse & TschUd, I977 (non publié) \\
\hline 3 & Brune des Alpes. & $\cdot$ & . . & & Suisse & TSCHUDI, I977 (non publié) \\
\hline 4 & Pie rouge allemande & & $\cdot$. & & Allemagne & RIECK et al. (1968) \\
\hline 5 & Brune allemande. & $\cdot$ & .. & & Allemagne & STRANZINGER et FöRSTER (I976) \\
\hline 6 & Red Poll . . . . & $\cdot$ & .. & & Grande-Bretagne & HARVEY (I976) \\
\hline 7 & British White. . . & . & 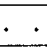 & & Grande-Bretagne & EIDDIDGE (I975) \\
\hline 8 & Rouge polonaise. & $\cdot$ & . . & & Pologne & SYSA, I977 (non publié) \\
\hline 9 & Simmental hongroise & & $\cdot$ & & Hongrie & Kovacs et PAPP, I977 (non publié) \\
\hline$\overline{10}$ & Pie rouge suédoise & . & . & & Suède, Hongrie & $\begin{array}{l}\text { GUSTAVSSON et RockBorN (I964) } \\
\text { Gustavsson, I969, Kovacs et } \\
\text { Gustavsson (I977) (non publié) }\end{array}$ \\
\hline I I & Rouge novvégienne & $\cdot$ & $\cdot$. & & Norgève & AMRUD (1969) \\
\hline 12 & Charolaise & $\cdot$ & . . & & $\begin{array}{l}\text { Grande-Bretagne, } \\
\text { France } \\
\text { Pologne, Roumanie }\end{array}$ & $\begin{array}{l}\text { HARVEY, I97r, FROGET et al. I972 } \\
\text { POPESCU, I973, QUEINNEC et al., I974 } \\
\text { SYSA, I977 (non publié), LIVESCU, I977 } \\
\text { (non publié) }\end{array}$ \\
\hline I3 & Limousine & . & . $\cdot$ & & $\begin{array}{l}\text { France, Grande- } \\
\text { Bretagne }\end{array}$ & $\begin{array}{l}\text { HARVEY, I972; POPESCU, I973; } \\
\text { QUEINNEC, 1974 }\end{array}$ \\
\hline I4 & Blonde d'Aquitaine & . & $\cdot \cdot$ & & $\begin{array}{c}\text { France, Nouvelle } \\
\text { Zélande }\end{array}$ & $\begin{array}{l}\text { QUEINNEC, I974; POPESCU, I974; } \\
\text { BRUERE et ChaPMAN, I973 }\end{array}$ \\
\hline I 5 & Montbéliarde. . . & $\cdot$ & $\cdot \cdot$ & & France & POPESCU, I97I, QUEINNEC, I972 \\
\hline I6 & Vosgienne... . & . & $\cdot \dot{ }$ & & France & POPESCU, 1976 \\
\hline I 7 & Gasconne... . & . & $\cdot \cdot$ & & France & QUEINNEC et al., 1974 \\
\hline$\overline{18}$ & Romagnole . . . & . & . . & & Italie & $\begin{array}{l}\text { RUGIATTI et FEDRIGO, I967 } \\
\text { DE GIOVANNI A. (I976) }\end{array}$ \\
\hline I9 & Chianina . . . & . & $\cdot \cdot$ & & Italie & DE GIovannI, 1976 \\
\hline 20 & Marchigiana . . & . & $\cdot \cdot$ & & Italie & DE GIOVANNI, I977 (non publié) \\
\hline $2 \mathrm{I}$ & Modicana. . . . & . & . & & Italie & DE GIOvanNI, I977 (non publié) \\
\hline 22 & Mucca Pisana. . & . & $\cdot \cdot$ & & Italie & SALERNO, I977 (non publié) \\
\hline 23 & Friesian . . . . & . & $\cdot \cdot$ & & U.S.A. & HERSCHLER et FECHHEIMER, I966 \\
\hline 24 & Guernsey · · · · & . & $\cdot \cdot$ & & Canada & BONGSO J., BASRUR, I966 \\
\hline 25 & Holstein $\times$ Criollo & - & $\cdot$ & & Cuba & BE'TANCOURT et al., I974 \\
\hline 26 & Thaï . . · · · & $\dot{ }$ & $\cdot \cdot$ & & Thaillande & FISCHER (I97I) \\
\hline 27 & Brune de l'Atlas. & . & $\cdot$ & & Maroc & FISCHER, (I975) \\
\hline 28 & Baoulé . . . . . & . & $\cdot$ & & Côte d'Ivoire & POPESCU, I977 (non publié) \\
\hline
\end{tabular}




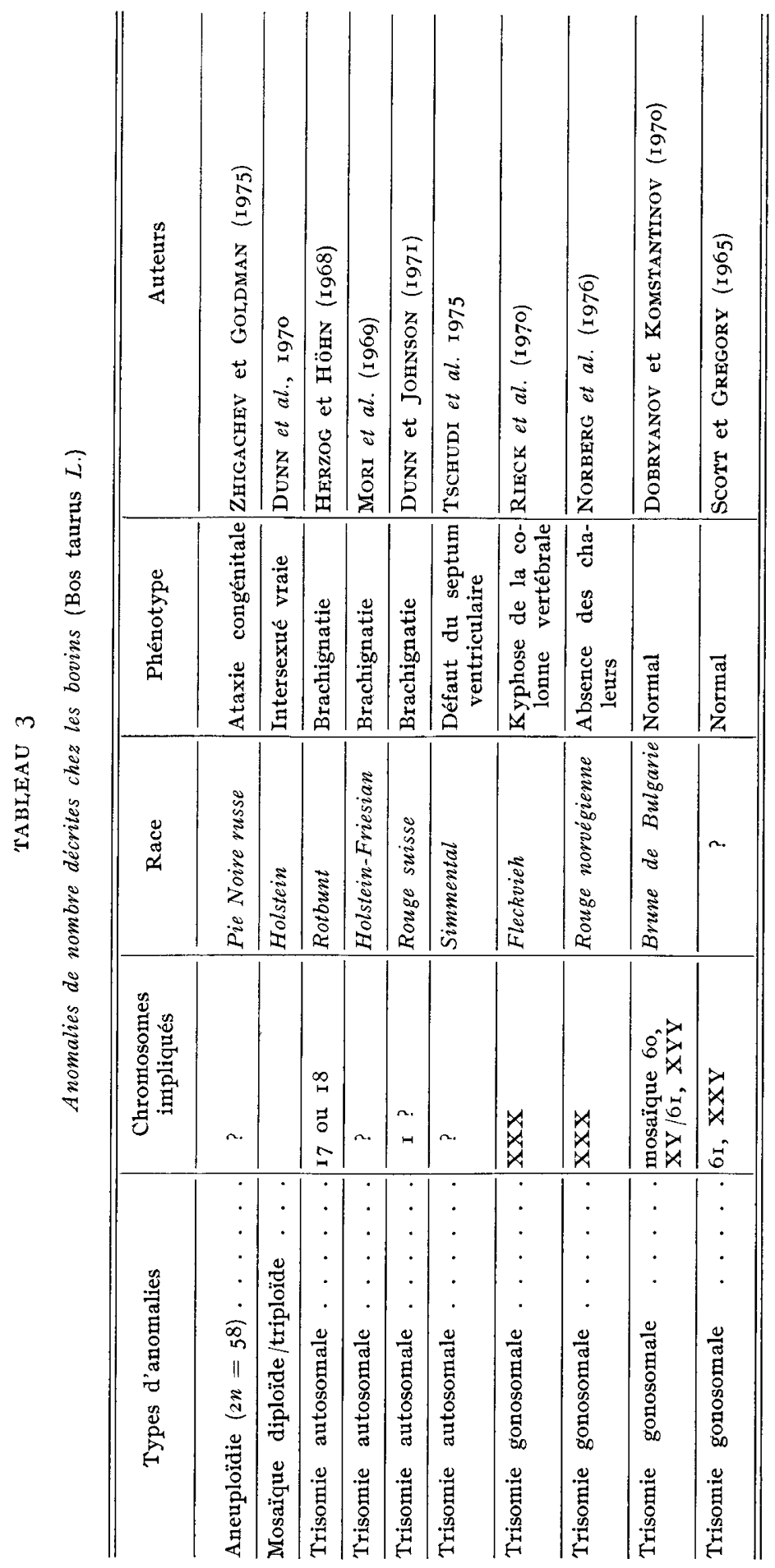


Le nombre d'animaux étudié varie considérablement d'une race à l'autre, car la seule Rouge suédoise représente un tiers du nombre total d'animaux à caryotype connu. Les différences sont aussi importantes si l'on compare différentes régions géographiques. En effet, la plupart des animaux étudiés, I I 0oo, se trouvent en Europe, alors que pour 1'Amérique du Nord et 1'Australie on enregistre respectivement environ I ooo animaux étudiés et 37 seulement pour 1'Afrique.

\section{Les anomalies de structure}

Le tableau I donne la liste des anomalies de structure. On notera tout d'abord que la translocation robertsonienne est l'anomalie la plus fréquente chez cette espèce. Elle peut impliquer divers chromosomes du complément, mais celle qui est la plus répandue et qui atteint les fréquences les plus élevées, concerne les chromosomes I et 29. Elle a été à ce jour identifiée dans 28 races, réparties en Europe, Amérique du Nord et Centrale, Asie et Afrique (tabl. 2). Il faut noter en outre, que sur 37 animaux seulement étudiés en Afrique, deux animaux, appartenant à deux races différentes, ont été trouvés porteurs de cette translocation I/29 (FISCHER et al., I975, Popescu, I977, non publié). On remarquera cependant que l'homologie de 1'anomalie dans toutes les races citées n'est pas établie, car dans peu de cas les caryotypes des animaux porteurs ont été étudiés par les méthodes des bandes. Les risques de confusion dans un caryotype dont tous les autosomes ont une morphologie semblable, sont grands (POPESCU, 1969) et il est possible que dans certains cas, un autre chromosome que le I ou le 29 soit impliqué dans la fusion.

L'hypothèse d'une origine commune et ancienne de cette anomalie exprimée antérieurement (Gustavsson, I969, POPESCU, I97I) semble appuyée par sa large distribution géographique. De plus, dans tous les cas où la méthode des bandes $\mathrm{C}$ a été appliquée sur des animaux de races différentes porteurs de l'anomalie, elle a toujours révélé un chromosome fussionné monocentrique, possédant un seul bloc d'hétérochromatine constitutive péricentromérique (POPESCU, I973, I974, Gustavsson, 1976, BLAZAK et Ei_DRIDGE, 1977). Or, l'on considère que les chromosomes fusionnés monocentriques, contrairement aux dicentriques, sont plus stables, et de ce fait, plus anciens (NIEBUHR, I973).

\section{Les anomalies de nombre}

Les anomalies de nombre ayant des effets délétéres importants sont souvent léthales, ce qui explique leur fréquence réduite par rapport aux anomalies de structure (tab1. 3).

A part quelques cas de trisomie gonosomale, qui provoque des troubles de reproduction, on remarque surtout une trisomie autosomale, trouvée dans des régions différentes, mais accompagnée toujours par les mêmes malformations anatomiques très caractéristiques (HERzoG et al., I968, MorI et al., I969).

\section{Conclusions}

Le développement de la cytogénétique des animaux domestiques ces dernières années, en particulier chez les bovins, se traduit par l'augmentation du nombre d'animaux étudiés et par la découverte de nouvelles anomalies. D'autre part, 
l'adaptation chez les animaux domestiques de la plupart des techniques de bandes utilisées chez l'homme, devrait conduire à une meilleure connaissance des caryotypes de ces espèces et résoudre le problème de l'identification des chromosomes impliqués dans différentes anomalies. La conférence de Reading (I976) constitue un premier effort dans la réalisation d'un idiogramme standard " à bandes " pour chaque espèce domestique. Disposant de ces méthodes d'analyse affinées, il serait souhaitable que chaque type d'anomalie soit étudié par ces méthodes, pour définir avec précision les chromosomes impliqués. Ceci pourrait servir également à confirmer l'homologie d'une anomalie présente dans différentes races et régions géographiques.

Les caractéristiques des chromosomes bovins, à savoir leur nombre élevé et la morphologie identique des autosomes explique pourquoi, malgré 1'amélioration considérable des méthodes d'identification, il subsiste encore beaucoup d'incertitude dans l'identification de certaines anomalies.

Il serait aussi important que les études des chromosomes mitotiques soier, $t$ complétées par des études sur la meïose. L'analyse des différents stades méiotiques, tels la diacynèse ou la métaphase II, constitue en effet, une voie d'approche dans la connaissance du mode de ségrégation et des types gamétiques qui en résultent, chez les hétérozygotes pour une anomalie de structure.

Reçu pour publication en décembre 1977.

\section{Remerciements}

Nous remercions tous les auteurs participants au IIre Colloque de Cytogénétique qui ont bien voulu nous envoyer les résultats concernant les anomalies chromosomiques identifiées dans leur Laboratoire.

\section{Summary \\ Chromosomal abnormalities in cattle (Bos taurus $\mathrm{I}_{1}$. ). Present statement of knowledge}

Combining the data from the literature and from the participants the $3^{\text {th }}$ Cytogenetics Conference, an inventory of the animals and the chromosomal abnormalities in cattle was carried out. Over I 3 oco animals have been studied in a cytogenetical point of view. The tables present the structural abnormalities (Table I) number abnormalities (Table 3 ) and the list of 28 breeds carrier of the I/29 translocation.

\section{Références bibliographiques}

AMRUd J., 1969. Centric fusion of chromosomes in Norvegian red cattle (N.R.F.). Hereditas, 62, 293-302.

Betancourt A., Gutierrez C., SANChez Aida, I974. Importancia del estudio chromosomico in bovinos selectionados como reproductoves. Ist World Congress on Genetics applied to livestock production Madrid, 7-I I oct., 3, 2 I3-2 18.

BLAZAK W. F., ELDRIDGE F. E., I977. A Robertsonian translocation and its effect upon fertility in Brown Swiss cattle. J. Dairy Sci., 60, I I33-1 I42.

BONGSO A., BASRUR P. K., I976. Chromosome anomalies in canadian guernsey bulls. Cornell Vet., 66, 476-489:

Bruere A. N., Chapman H. M., 1973. Autosomal translocations in two exotic breeds of cattle in New Zealand. Vet. Rec., 92, 6r5-620. 
Cribiu E. P., Popescu C. P., I974. Un cas de chromosomes Y anormalement long chez Bos taurus L. Ann. Genet. Selec. Anim., 6, 387-390.

Darke R., Berland H. M., GUennec G., I974. Une nouvelle translocation robertsonienne chez les bovins. Ann. Génét. Sél. anim., 6, 297-303.

DobRiYanov D., Konstantinov G., I970. A case of mosaicism of the $5^{8} \mathrm{AXY}, 5^{8} \mathrm{AXYY}$ type in a male calf of the Bulgarian Brown cattle breed. C.R. Acad. Sci. agric., Bulg. 3, 27I276.

DunN H. O., Johnson R. H., 1972. A 6IXY cell line, in a calf with extreme brachygnathia. J. of Dairy Sci., 55, 524-526.

ELDRIDGE F. E., I974. A dicentric Robertsonian translocation in a dexter cow. J. Hered., $65,353-355$

ELDRIDGE E. F., I975. High frequency of a Robertsonian translocation in on herd of British White cattle. Vet. Rec., 97, 71-72.

FechHeimer N. S., r973. A cytogenetic survey of young bulls in the U.S.A. Vet. Rec., 93 (20), 535-536.

Fischer H., Höhn, Henni, ScheurmanN, Elike, I975. Unternchungen über die karyotypen des Braunen Atlas Rindes. Giessener Beitr. Erbpath. Zuchthyg., 6, 70-79.

Froget J., Coulon J., Main M. C., Daubiez J. M., 1972. Anomalie chromosomique de type fusion centrique chez un veau Charolais. Bull. Soc. Sci. Vet. et Med. comparée (Lyon), 74, I3I-I35.

Gustavsson I., RockBorn G., 1964. Chromosome abnormality in three cases of lymphatic leukaemia in cattle. Nature (Lond.), 203, 990.

Gustavsson, Fraccaro M., Tiepolo L., Lindstein J., i968. Presumptive-X-autosome translocation in a cow: preferential inactivation of the normal $\mathrm{X}$ chromosome. Nature, 218, 183 I84.

Gustavsson I., I969. Cytogenetics, distribution and phenotypic effects of a translocation in swedish cattle. Heveditas, 63, 68-I69.

Gustavsson I., Hageltorn M., ZeCH L., I976. Identification of the $1 / 29$ translocation in the Swedish Red and White (SRB) cattle breed by utilization of new staining techniques. Hereditas, 82, 260-262.

Hansen K. M., I969. Bovine tendem fusion and infertility. (Abstr.). Hereditas, 63, 453-454

HARvey M. J. A., I972. Chromosomes analysis of cattle in Great Britain. Proc. VIII Internat. Congr. Anim. Reprod. Artif. Insent. Munich I Ior-IIo3.

HARVEY J. A., I974. Chromosome analysis of cattle populations. Vet. Rec., 94, 227.

HARVEY M. J. A., I976. Veterinary cytogenetics. Vet. Rec., 98 (24), 479-481.

HeRzoG A., HöHN H., I968. Autosomal Trisomie bei einem kalb mit Brachygnatia inferior and Ascites congenitas. Dtsch. Tievaerztl. wochenschr., 23, 604-605.

HERZOG A., HOHN H., I97I. Zytogenetische befunde bei angeborenen anomalien des zentralnervensystems des rind. Ann. Génét. Sél. anim., 3, 225-234.

HeRsChlER M. S., FechHeIMER N. S., r966. Centric fusion of chromosomes in a set of bovine triplets. Cytogenetics, 5, 307-3I2.

Kovacs A., Meszaros I., Sel. yei M., Vass L., I973. Mosaic centromeric fusion in a HolsteinFriesian bull. Acta Biol., 24, 2 I5-220.

Mori M., SASA Ki M., Makino S., Ishikawa T., KawaTA K., I969. Autosomal trisomy in a malformed new born calf. Proc. Japan Acad., 45, 955-959.

Norberg H. S., Refsdal. A. O., Garm O. N. et al., r976. A case report on X-trisomy in cattle. Hereditas, 82, 69-72.

POLLOCK D. I., I972. A chromosome abnormality in Friesian Cattle in Great Britain. Vet. Rec., 90, 309-310

Popescu C. P., 1971. Deux cas nouveaux de fusion centrique chez les bovins. Ann. Génét. Sél. anim., 3, 521-526.

Popescu C. P., I972. Un cas possible d'inversion péricentrique chez les bovins. Ann. Génét., 15, I97-200.

Popescu C. P. (a), I973. L'hétérochromatine constitutive dans le caryotype bovin normal et anormal. Ann. Génét., 16, 183-188.

Popescu C. P., I973 (b). Nouvelles observations sur une fusion centrique chez Bos taurus L. Ann. Génét. Sél. anim., 5, 435-440.

PoPEscu C. P., I974 (a). Étude du caryotype bovin par une nouvelle méthode cytogénétique : les bandes C. I er Congr. Mond. Génét. appl. l'élev., Madrid 7-I I octobre I974, 3, I59-I64.

Popescu C. P., $1974(b)$. Observations sur une fusion centrique chez les bovins (Bos taurus L.). $I^{\mathrm{er}}$ Congv. Mond. Génét. appl. l'élev., Madrid 7-I I octobre 74, 3, I65-168. 
POPESCU C. P., 1975. Sur la fréquence de la translocation $\mathrm{I} / 29$ dans certaines races bovines. In $z$ Europ Kolloq Zytogenet. (Chromosomenpath.) in veterinarmed saugetier. Giessen, I975, 277-282.

Popescu C. P., I976. New data on pericentric inversion in cattle (Bos taurus L.). Ann. Génét. Sél. anim., 8, 443-448.

Popescu C. P., I 977 . A new type of Robertsonian translocation in cattle. J. Hered., 68, I38-I 42.

Popescu C. P., Cribiu E. P., Tschudi P., 1976. Deux cas de fusion centrique chez Bos tanrus I. en Suisse. Ann. Génét. Sél. anim., 7, 317-319.

Queinnec G., Darre R., Berland H. M., Raynaud J. C., I974. Étude de la translocation I-29 dans la population bovine du Sud-ouest de la France : conséquences zootechniques. $I^{\text {er }}$ Cong. Mond. Génétique appl. Elev., Madrid, 3, I3I-I5I.

REFSDAL, A. O., I976. Low fertility in daughters of bulls with I/29 translocation. Acta Vet. Scand., 17, I90-I 95 .

RiEck G. W., HöhN H., Herzog A., I968. Familial occurence of centromeric chromosome fusion in cattle. Zuchthygiene, 3, I I 7-I82.

RIEck G. W., Hohn H., HERzoG A., x970. X-Trisomie beim Rind mit Anzeichen familiarer Disposition fur Meiosestörungen. (All.) Rés. Angl. Cytogenetics, 9, 40I-409.

RUGIATI S., FEDRIGO M., I967. Alterazione cromosomica riscontrata in un toro acondroplasico di razza Romagnola. L'Atenco Parmense. Acta Bio. Medica, 38, 3-7.

STRANZINGER G. F., FöRSTER M., I976. Autosomale Chromosomentranslokationen beim Fleckund Braunvieh. (All.) Res : Angl. Experientia, 32, 24-27.

Short R. V., Smith, Janet, Mann T., Evans E. V., Hallet, Janet, Fryer, Anthea, HamerTON L. J., 1969. Cytogenetic and endocrine studies of a freemartin heifer and its bull co-twin. Cytogenetics, 8, 369-388.

Succi G., Giovanis A. de, Molteni L., r 976 . Nouvelles observations sur une translocation robertsonienne en race Romagnole. Ann. Génét. Sél. anim., 8, 37-40.

Scott C. D., GRegory P. W., I965. An XXY trisomic in an intersex of Bos taurus (abstr.). Genetics, 52, 473-474.

TSCHUdi F., UeltSchi G., MarTig J. et al., i975. Autosomal trisomy as the cause of a defect of the interventricular septum in a Simmental calf. (All.). Schweiz. Arch. Tierheilk., 117, 335340 .

Zhigachev A. I., Golddman I. L., 1975. Congenital ataxia in a Black Pied heifer. Dokl. Vses. Akad. S-kh. Nauk, 3, 36-37. 